\title{
Effects of arbuscular mycorrhiza and composted market waste on the performance of Tiannug 1 variety of kenaf (Hibiscus cannabinus Linn.)
}

\author{
Babatunde SAHEED BADA $^{1 *}$ and Olajire FAGBOLA ${ }^{2}$ \\ ${ }^{I}$ Department of Environmental Management and Toxicology, Federal University of Agriculture, \\ Abeokuta, Ogun State, Nigeria. \\ ${ }^{2}$ Department of Agronomy, University of Ibadan, Ibadan, Nigeria. \\ *Corresponding author, E-mail: bsbada2000@yahoo.com; Tel: +2348037250964
}

\begin{abstract}
This study determined effects of composted market waste and Arbuscular Mycorrhiza (AM) on Root Colonization (RC) and fibre yield of kenaf. The experimental design was $2 \times 12$ factorial in a Completely Randomized Design replicated three times. The treatments were with $\mathrm{AM}\left(\mathrm{AM}^{+}\right)$and without $\left(\mathrm{AM}^{-}\right)$and twelve levels of soil amendment: 0, NPK 20:10:10 (60 $\left.\mathrm{kg} \mathrm{N} \mathrm{ha}^{-1}\right)$, purely composted market waste (20, 40, 60, 80 and $\left.100 \mathrm{~kg} \mathrm{~N} \mathrm{ha}^{-1}\right)$, and composted market waste fortified with superphosphate and urea (20, 40,60, 80 and $100 \mathrm{~kg} \mathrm{~N} \mathrm{ha}^{-1}$ ). Residual effect of the treatments was also determined. Data on RC and yield were collected and analyzed using descriptive statistics and ANOVA. RC ranged from 14.4 to $78.1 \%$. Inoculated Tiannug 1 at 40 $\mathrm{kg} \mathrm{N} \mathrm{ha}{ }^{-1}$ composted market waste fortified with superphosphate and urea had significantly $(\mathrm{p}<0.05)$ higher RC $(79.8 \%)$, bast $\left(9.6 \mathrm{~g} \mathrm{pot}^{-1}\right)$ and core $\left(19.9 \mathrm{~g} \mathrm{pot}^{-1}\right)$ yields. For the residual effect, inoculated Tiannug 1 at $100 \mathrm{~kg} \mathrm{~N} \mathrm{ha}^{-1}$ purely composted market waste had significantly $(\mathrm{p}<0.05)$ higher RC $(69.0 \%)$, bast $\left(2.7 \mathrm{t} \mathrm{ha}^{-1}\right)$ and core $\left(5.8 \mathrm{t} \mathrm{ha}^{-1}\right)$ yields. Tiannug 1 was highly colonized by AM and the optimum yields observed at $40 \mathrm{~kg}$ $\mathrm{N}^{-1}$ composted market waste fortified with superphosphate and urea.

(c) 2014 International Formulae Group. All rights reserved.
\end{abstract}

Keywords: Arbuscular mycorrhiza, composted market waste, kenaf, performance.

\section{INTRODUCTION}

Continuous use of inorganic fertilizer results to soil acidification (Bada et al., 2012). Organic manures are excellent sources of organic matter but relatively low in nutrients for example nitrogen, phosphorus and potassium (Bada et al., 2012). Complementary use of organic manure and inorganic fertilizer ensures the availability of nutrient throughout the growth period of crops. The use of composted market waste for crop production is gaining popularity worldwide because it is environmentally friendly, cheaper and easily available compared to inorganic fertilizer. In view of this, complimentary use of low chemical inputs and organic manure may be a cost effective economic strategy (Omueti et al., 2000). As a way of reducing total dependence on the use of fertilizer, an integrated fertility management system focusing on biological approach, which is ecofriendly and less expensive, is desirable.

Mycorrhizal symbiosis is well recognized as a biological tool to enhance 
nutrient acquisition in most plants growing on deficient soils (Cardoso and Kuyper, 2006). Mycorrhizal is a symbiotic association between plant root and specialized soil fungi with evidence that it helps plants in nutrient acquisition of immobile nutrients such as $\mathrm{P}$, $\mathrm{N}, \mathrm{Zn}$ and $\mathrm{Cu}$ in deficient soils (Clark and Zeto, 2000; Hodge, 2003; Dare et al., 2008; Ibiremo and Fagbola, 2008).

Kenaf (Hibiscus cannabinus L.) stem produces two types of fibre, a coarser fibre in the outer layer (bast) and a finer fibre in the inner (core). Uses of kenaf include fibre and food (Bert, 2002; Zhang, 2003), medicine (Cheng, 2001), medium for mushroom cultivation (Cheng, 2001; Liu, 2003), phytoremediation (Bada and Kalejaiye, 2010; Bada and Raji, 2010; Bada and Umunnakwe, 2011), oil and chemical absorbents (Sameshima, 2000). In addition, the bast fibre can be converted to pulp for newsprint, hydrocarbon free bags, ropes and textiles (Kuchinda and Ogunwole, 2000; Webber et al., 2002). Kenaf fibre is used in making ropes, sack and other domestic purposes such as creating fences and thatching for dwelling (IART, 2010). Kenaf generally gives the greatest response to nitrogen, followed by phosphorus (Wilson, 2003; IART, 2010). Therefore, for sustainable kenaf production in a nutrient degraded soil, there is need to study effect of arbuscular mycorrhizal inoculation and composted market waste application on the growth and yield of kenaf. This will go a long way in reducing total dependence on the use of inorganic fertilizer and also reduce the problem of waste management at little or no cost. The objectives of this study were to investigate the effect of composted market waste application on the root colonization of kenaf by Arbuscular Mycorrhiza (AM); evaluate the effect of AM with composted market waste on growth and yield of kenaf and examine the residual effect of AM inoculation and composted market waste on bast and core yield of kenaf.

\section{MATERIALS AND METHODS}

The screenhouse experiment was carried out at the Institute of Agricultural Research and Training (IART) Moor Plantation, Ibadan on Latitude $7^{\circ} 22.5^{\prime} \mathrm{N}$ and Longitude $3^{\circ} 50.5^{\prime} \mathrm{E}$ in the rainforest of SouthWestern Nigeria.

\section{Description of the planting materials}

Two types of market waste-based fertilizers (produced by Pacesetter Fertilizer Company, Ibadan, Nigeria) namely: composted market waste fortified with superphosphate and urea (Pacesetter Grade A fertilizer) and purely composted market waste without any additive (Pacesetter Grade B fertilizer), prior to application, were taken to the laboratory for proximate analysis. However, N.P.K. 20:10:10 fertilizer at recommended rate of $60 \mathrm{~kg} \mathrm{~N} \mathrm{ha}{ }^{-1}$ (IART, 2010) was also used. Mycorrhizal inoculum (Glomus mosseae) consisting of chopped roots of the trapping plant, hyphae, spores and soil was collected from the Soil Microbiology Laboratory, Department of Agronomy, University of Ibadan, Ibadan, Nigeria. In an experiment carried out in the same area, Atayese et al. (1993), reported that of all the AM present, Glomus mosseae is the most prominent / abundant. As a result, Glomus mosseae was used in this study. Tiannug 1, one of the highly yielding varieties of kenaf suitable for different agro-ecological zones in Nigeria (IART, 2010) was collected from IART, Ibadan, Nigeria.

\section{Experimental design}

A $2 \times 12$ factorial experiment in a Completely Randomized Design with three replicates was carried out to assess the effects of two levels of mycorrhiza (with and without) and twelve levels of fertilizers: 0, NPK 20:10:10 (60 kg $\left.\mathrm{N} \quad \mathrm{ha}^{-1}\right)$, purely composted market waste $(20,40,60,80$ and $100 \mathrm{~kg} \mathrm{~N} \mathrm{ha}{ }^{-1}$ ) and composted market waste fortified with superphosphate and urea (20, 40, 60, 80 and $100 \mathrm{~kg} \mathrm{~N} \mathrm{ha}^{-1}$ ) on the root 
colonization, growth and fibre yield of Tiannug 1.

Soil sample collection, preparation, sowing and data collection

Representative topsoil $(0-15 \mathrm{~cm})$ samples were collected from research farm of IART, Ibadan and thoroughly mixed together. Sub-samples were taken to determine the physical and chemical properties using the methods described in Chopra and Kanwar (1999). Arbuscular mycorrhiza infective propagule was also determined using the 'Most Probable Number' (MPN) technique (Porter 1979). Each pot contained $10 \mathrm{~kg}$ soil. Composted market waste fortified with superphosphate and urea $(20,40,60,80$ and $100 \mathrm{~kg} \mathrm{~N} \mathrm{ha}^{-1}$ ) and purely composted market wastes $(20,40,60,80$ and $100 \mathrm{~kg} \mathrm{~N}$ ha-1) were thoroughly mixed with the soil in the pots 24 hours before planting. Sixty $\mathrm{kg} \mathrm{N}^{-1}$ of N.P.K. 20:10:10 fertilizer was applied the third week after planting by side placement. Twenty grams of mycorrhizal inoculum (Glomus mosseae) was applied per pot of those designated as inoculated. The method of application was by filling the pots threequarter way and then evenly spread the mycorrhiza inoculum on it (Carling et al., 1978). The pots were then filled with the rest of the soil and watered. Seeds of Tiannug I were sown at the rate of $4-6$ seeds per pot. After germination, the plants were thinned to one per pot and left to grow in the pot for three and half months (14 weeks). Plant height was measured using metre rule and stem diameter $(\mathrm{cm})$ using vernier caliper. After harvesting, bast and core yield were determined using weighing balance. Percentage root colonization was determined using the method described by Giovanneti and Mosse (1980).

\section{Assessment of residual effects of AM and fertilizers}

This was carried out to determine the residual effect of arbuscular mycorrhiza, purely composted market waste, composted market waste fortified with superphosphate and urea, and $\mathrm{N}: \mathrm{P}: \mathrm{K}$ fertilizer on the root colonization, growth and yield of Tiannug 1 .

\section{Soil preparation and sowing}

After harvesting, the soil in each pot was thoroughly mixed. Neither fertilizer nor mycorrhiza was applied and kenaf seeds were then sowed

\section{Statistical analyses}

Data were analyzed using descriptive statistics (mean) and analysis of variance. Standard error was used to separate the means at $\mathrm{p}<0.05$.

\section{RESULTS \\ Soil characteristics and proximate analysis of fertilizers}

The soil used in the screenhouse experiment was sandy loam in texture, with a $\mathrm{pH}$ of 6.1 (Table 1). Also, the soil organic matter, total nitrogen, available phosphorus and exchangeable potassium were $15.0 \mathrm{~g} \mathrm{~kg}^{-1}$, $1.5 \mathrm{~g} \mathrm{~kg}^{-1}, 2.1 \mathrm{mg} \mathrm{kg}^{-1}$ and $0.19 \mathrm{cmol} \mathrm{kg}^{-1}$ respectively. Arbuscular mycorrhiza infective propagule density was 110 infective propagules per $100 \mathrm{~g}$ soil.

$$
\begin{array}{rrrr} 
& \text { Composted Market Waste } & \text { Fortified } \\
\text { with } & \text { Superphosphate } & \text { and } & \text { Urea }
\end{array}
$$
(CMWFWSAU) had higher concentrations of primary nutrient such as nitrogen, phosphorus and potassium than Purely Composted Market Waste (PCMW) (Table 2).

\section{Effects of AM and fertilizers on the growth of Tiannug 1}

Arbuscular mycorrhiza inoculation and composted market waste application rates significantly affected the stem diameter of Tiannug 1(Table 3). Inoculated Tiannug 1 at 0 fertilizer level had higher stem diameter than non-inoculated counterpart at 0 fertilizer level throughout the growth period. Increase in the levels of PCMW and CMWFWSAU without arbuscular mycorrhizal inoculation resulted in the significant $(\mathrm{p}<0.001)$ increase in stem diameter from 20 to $60 \mathrm{~kg} \mathrm{~N}^{-1}$ of both PCMW and CMWFWSAU (Table 3). Without mycorrhizal inoculation, significantly ( $\mathrm{p}<0.001)$ higher stem diameter was observed at $60 \mathrm{~kg} \mathrm{~N} \mathrm{ha}^{-1}$ of CMWFWSAU. On the effects of arbuscular mycorrhizal inoculation and fertilizers application, stem diameter of inoculated Tiannug 1 was 
significantly $(\mathrm{p}<0.001)$ higher than the noninoculated counterpart at $60 \mathrm{~kg} \mathrm{~N} \mathrm{ha}{ }^{-1}$ of NPK, $40 \mathrm{~kg} \mathrm{~N}^{-1}$ of PCMW, 40 and $60 \mathrm{~kg} \mathrm{~N}$ $\mathrm{ha}^{-1}$ of CMWFWSAU from 6 to 14WAP (Table 3). Significantly $(p<0.001)$ higher stem diameter was observed at $40 \mathrm{~kg} \mathrm{~N} \mathrm{ha}^{-1}$ of CMWFWSAU 14WAP.

Mycorrhizal inoculation significantly $(p<0.001)$ increased plant height at 0 fertilizer level compared to their noninoculated counterpart also at 0 fertilizer level (Table 4). Among the various fertilizer levels without mycorrhizal inoculation, $60 \mathrm{~kg} \mathrm{~N}^{-1}$ of CMWFWSAU had significantly ( $p$ < 0.001) higher plant height compared to other levels of composted market waste without mycorrhizal inoculation (Table 4). On the effects of mycorrhizal inoculation and composted market waste application rates, inoculated Tiannug 1 had significantly ( $\mathrm{p}<$ $0.001)$ higher plant height than non-inoculated counterpart at $60 \mathrm{~kg} \mathrm{~N} \mathrm{ha}^{-1}$ of NPK, $40 \mathrm{~kg} \mathrm{~N}$ $\mathrm{ha}^{-1}$ of PCMW, 40 and $60 \mathrm{~kg} \mathrm{~N} \mathrm{ha}{ }^{-1}$ of CMWFWSAU from 6 to 14WAP. Significantly $(\mathrm{p}<0.001)$ higher plant height was observed in the inoculated Tiannug 1 at $40 \mathrm{~kg} \mathrm{~N} \mathrm{ha}^{-1}$ of CMWFWSAU.

\section{Influence of AM and fertilizers on the colonization and yields of Tiannug 1}

Inoculated Tiannug 1 at 0 fertilizer application had significantly ( $\mathrm{p}<0.001)$ higher bast yield than their non-inoculated counterpart at 0 fertilizer application (Table 5). Increase in the levels of PCMW and CMWFWSAU without mycorrhizal inoculation significantly $(\mathrm{p}<0.001)$

increased the bast yield from 0 to $60 \mathrm{~kg} \mathrm{~N} \mathrm{ha}^{-1}$ while at $80 \mathrm{~kg} \mathrm{~N} \mathrm{ha}^{-1}$ or more, a decline in bast yield occurred (Table 5). On the other hand, inoculated Tiannug 1 had significantly $(\mathrm{p}<0.001)$ higher bast yield than the noninoculated counterpart at $60 \mathrm{~kg} \mathrm{~N}$ ha $^{-1}$ of NPK, $40 \mathrm{~kg} \mathrm{~N} \mathrm{ha}^{-1}$ of PCMW, 40 and $60 \mathrm{~kg} \mathrm{~N}$ $\mathrm{ha}^{-1}$ of CMWFWSAU. The highest bast yield was observed at $40 \mathrm{~kg} \quad \mathrm{~N} \quad \mathrm{ha}^{-1}$ of CMWFWSAU (Table 5).Without mycorrhizal inoculation, significantly $(\mathrm{p}<0.001)$ higher core yield was observed at $60 \mathrm{~kg} \mathrm{~N} \mathrm{ha}^{-1}$ of CMWFWSAU (Table 5).
Fertilizers application with mycorrhizal inoculation had significantly ( $\mathrm{p}<0.001$ ) higher percentage mycorrhizal colonization than fertilizers application without mycorrhizal inoculation at $40 \mathrm{~kg} \mathrm{~N}^{-1}$ of PCMW and CMWFWSAU.

\section{Residual effects of AM and fertilizers on the growth \\ Stem diameter of the inoculated} without fertilizer application was higher than the stem diameter of the non-inoculated without fertilizer application 14WAP (Table 6). Stem diameter of the non-inoculated significantly $(\mathrm{p}<0.001)$ increased as the levels of PCMW and CMWFWSAU increased from 20 to $100 \mathrm{~kg} \mathrm{~N}^{-1}$ from 6WAP to 14WAP. Significantly $(\mathrm{p}<0.001)$ higher stem diameter was observed between the inoculated and the non-inoculated at 40 and $100 \mathrm{~kg} \mathrm{~N}^{-}$ 1 of PCMW; and $40 \mathrm{~kg} \mathrm{~N} \mathrm{ha}{ }^{-1}$ of CMWFWSAU 14WAP (Table 6). Significantly $(\mathrm{p}<0.001)$ higher stem diameter was observed in the inoculated at $100 \mathrm{~kg} \mathrm{~N}$ ha ${ }^{1}$ of PCMW 14WAP. However, plant height of the inoculated without fertilizer application was $12.7 \%$ higher than the plant height of the non-inoculated without fertilizer application (Table 7). The higher the levels of PCMW and CMWFWSAU applied without mycorrhizal inoculation, the higher were the residual effect on the plant height with $100 \mathrm{~kg}$ $\mathrm{N} \mathrm{ha}^{-1}$ of PCMW having significantly ( $\mathrm{p}<$ 0.001) higher plant height 14WAP (Table 7). The percentage range of 3.5 to $9.0 \%$ was observed between the inoculated and noninoculated with fertilizers application 14WAP.

\section{Residual effects of AM and fertilizers on the colonization and yield of Tiannug 1 \\ Core yield and mycorrhizal} colonization were significantly $(\mathrm{p}<0.05)$ affected by the residual effects of composted market waste application rates and mycorrhizal inoculation (Table 8). Inoculated Tiannug 1 at 0 fertilizer level was higher than the non-inoculated counterpart at the same fertilizer level by $10.6 \%$. Increase in the levels of PCMW and CMWFWSAU without mycorrhizal inoculation resulted in the significant $(p<0.001)$ increase in bast yield at 
$100 \mathrm{~kg} \mathrm{~N} \mathrm{ha}^{-1}$ of PCMW and CMWFWSAU. Inoculated Tiannug 1 had significantly ( $\mathrm{p}<$ 0.001) higher bast yield than the noninoculated counterpart at 80 and $100 \mathrm{~kg} \mathrm{~N} \mathrm{ha}^{-1}$ of PCMW, and also at $100 \mathrm{~kg} \mathrm{~N} \mathrm{ha}^{-1}$ of CMWFWSAU (Tables 8). On the residual effect of PCMW and CMWFWSAU with mycorrhiza inoculation, significant $(\mathrm{p}<$ 0.001 ) increase were observed at 80 and 100 $\mathrm{kg} \mathrm{N} \mathrm{ha}{ }^{-1}$ of both PCMW and CMWFWSAU.

Inoculated Tiannug 1 at 0 fertilizer level was $22.1 \%$ higher than the noninoculated counterpart (Table 8). Significant $(\mathrm{p}<0.001)$ increase was observed up to $80 \mathrm{~kg}$ $\mathrm{N} \mathrm{ha}^{-1}$ of PCMW and CMWFWSAU. Significant increase was observed between the inoculated with fertilizer application and the non-inoculated counterpart at $100 \mathrm{~kg} \mathrm{~N} \mathrm{ha}^{-1}$ of PCMW and CMWFWSAU.

Inoculated was $7.2 \%$ higher than the non-inoculated (Table 8). Increase in the levels of PCMW and CMWFWSAU without mycorrhizal inoculation resulted in the significant $(\mathrm{p}<0.001)$ increased in the mycorrhizal colonization from 60 to $100 \mathrm{~kg} \mathrm{~N}$ ha $^{-1}$ of PCMW; and 80 to $100 \mathrm{~kg} \mathrm{~N} \mathrm{ha}^{-1}$ of CMWFWSAU. Inoculated Tiannug1 at 60, 80 and $100 \mathrm{~kg} \mathrm{~N} \mathrm{ha}^{-1}$ of PCMW and CMWFWSAU had significantly $(\mathrm{p}<0.001)$ higher mycorrhizal colonization than their non-inoculated counterpart at the same fertilizer level.

Table 1: Soil physical and chemical properties before planting.

\begin{tabular}{lc}
\hline Soil properties & Value \\
\hline Sand $\left(\mathrm{g} \mathrm{kg}^{-1}\right)$ & 760 \\
Silt $\left(\mathrm{g} \mathrm{kg}^{-1}\right)$ & 182 \\
Clay $\left(\mathrm{g} \mathrm{kg}^{-1}\right)$ & 58 \\
Textural class & Sandy loam \\
$\mathrm{pH}\left(\mathrm{H}_{2} \mathrm{O}\right)$ & 6.1 \\
Organic matter $\left(\mathrm{g} \mathrm{kg}^{-1}\right)$ & 15.0 \\
Total N $\left(\mathrm{g} \mathrm{kg}^{-1}\right)$ & 1.5 \\
Available P $\left(\mathrm{mg} \mathrm{kg}^{-1}\right)$ & 2.1 \\
$\left.\mathrm{~K}(\mathrm{cmol} \mathrm{kg})^{-1}\right)$ & 0.2 \\
$\mathrm{Ca}\left(\mathrm{cmol} \mathrm{kg}^{-1}\right)$ & 1.6 \\
$\mathrm{Na}(\mathrm{cmol} \mathrm{kg}$ & 0.6 \\
$\mathrm{Mg}\left(\mathrm{cmol} \mathrm{kg} \mathrm{kg}^{-1}\right)$ & 2.1 \\
Exch.acidity $(\mathrm{cmol} \mathrm{kg}$ & 0.1 \\
ECEC $\left(\mathrm{cmol} \mathrm{kg}^{-1}\right)$ & 4.6 \\
Base saturation $(\%)$ & 97.6 \\
\hline
\end{tabular}

Table 2: Proximate analysis of Composted Market Waste Fortified With Superphosphate And Urea (CMWFWSAU) and Purely Composted Market Waste (PCMW).

\begin{tabular}{lcc}
\hline Parameters & CMWFWSAU & PCMW \\
\hline Nitrogen $\left(\mathrm{g} \mathrm{kg}^{-1}\right)$ & 57.50 & 10.50 \\
Phosphorus $\left(\mathrm{mg} \mathrm{kg}^{-1}\right)$ & 4.48 & 0.95 \\
Potassium $\left(\mathrm{cmol} \mathrm{kg}^{-1}\right)$ & 3.05 & 1.00 \\
Cacium $\left(\mathrm{cmol} \mathrm{kg}^{-1}\right)$ & 1.25 & 2.12 \\
Magnesium $\left(\mathrm{cmol} \mathrm{kg}^{-1}\right)$ & 0.35 & 0.85 \\
\hline \multicolumn{2}{c}{ PCMW = Purely Composted } & Market Waste; CMWFWSAU = Composted Market Waste Fortified With \\
\multicolumn{2}{l}{ Superphosphate And Urea } &
\end{tabular}


Table 3: Effects of AM and fertilizers on the stem diameter $(\mathrm{cm})$.

\begin{tabular}{|c|c|c|c|c|c|c|}
\hline $\begin{array}{l}\text { Mycorrhiza } \\
\text { inoculation }\end{array}$ & Fertilizers application & 6 & 8 & 10 & 12 & 14 \\
\hline \multirow[t]{12}{*}{ With } & 0 & 0.40 & 0.47 & 0.54 & 0.65 & 0.71 \\
\hline & NPK & 0.82 & 0.95 & 1.08 & 1.15 & 1.22 \\
\hline & PCMW20 & 0.45 & 0.60 & 0.65 & 0.70 & 0.75 \\
\hline & PCMW40 & 0.62 & 0.75 & 0.80 & 0.90 & 1.00 \\
\hline & PCMW60 & 0.60 & 0.72 & 0.80 & 0.90 & 1.00 \\
\hline & PCMW80 & 0.55 & 0.65 & 0.75 & 0.85 & 0.90 \\
\hline & PCMW100 & 0.60 & 0.66 & 0.75 & 0.87 & 0.98 \\
\hline & CMWFWSAU20 & 0.50 & 0.62 & 0.68 & 0.75 & 0.78 \\
\hline & CMWFWSAU40 & 0.91 & 1.14 & 1.38 & 1.50 & 1.52 \\
\hline & CMWFWSAU60 & 0.85 & 0.95 & 1.10 & 1.18 & 1.40 \\
\hline & CMWFWSAU80 & 0.68 & 0.79 & 0.88 & 1.00 & 1.08 \\
\hline & CMWFWSAU100 & 0.74 & 0.88 & 0.95 & 1.06 & 1.10 \\
\hline \multirow[t]{12}{*}{ Without } & 0 & 0.30 & 0.44 & 0.50 & 0.58 & 0.66 \\
\hline & NPK & 0.75 & 0.90 & 1.00 & 1.07 & 1.11 \\
\hline & PCMW20 & 0.45 & 0.58 & 0.62 & 0.70 & 0.72 \\
\hline & PCMW40 & 0.54 & 0.64 & 0.70 & 0.78 & 0.82 \\
\hline & PCMW60 & 0.60 & 0.70 & 0.76 & 0.90 & 1.00 \\
\hline & PCMW80 & 0.55 & 0.65 & 0.72 & 0.80 & 0.85 \\
\hline & PCMW100 & 0.60 & 0.66 & 0.75 & 0.86 & 0.92 \\
\hline & CMWFWSAU20 & 0.50 & 0.61 & 0.65 & 0.70 & 0.75 \\
\hline & CMWFWSAU40 & 0.64 & 0.75 & 0.82 & 0.90 & 1.01 \\
\hline & CMWFWSAU60 & 0.78 & 0.95 & 1.05 & 1.10 & 1.20 \\
\hline & CMWFWSAU80 & 0.65 & 0.77 & 0.85 & 0.99 & 1.02 \\
\hline & CMWFWSAU100 & 0.71 & 0.84 & 0.92 & 1.05 & 1.08 \\
\hline \multicolumn{7}{|l|}{ SEM } \\
\hline Mycorrhiza (M) & & 0.0001 & 0.0032 & 0.0032 & 0.0025 & 0.0034 \\
\hline Fertilizers (F) & & 0.0001 & 0.0079 & 0.0079 & 0.0062 & 0.0082 \\
\hline \multicolumn{7}{|l|}{ Interaction } \\
\hline$M \times F$ & & 0.0001 & 0.0112 & 0.0112 & 0.0088 & 0.0116 \\
\hline \multicolumn{7}{|l|}{ ANOVA } \\
\hline M & & $* * *$ & $* * *$ & $* * *$ & $* * *$ & $* * *$ \\
\hline $\mathrm{F}$ & & $* * *$ & $* * *$ & $* * *$ & $* * *$ & $* * *$ \\
\hline \multicolumn{7}{|l|}{ Interaction } \\
\hline$M \times F$ & & $* * *$ & $* * *$ & $* * *$ & $* * *$ & $* * *$ \\
\hline $\begin{array}{l}\text { Values are means of } \\
6,8,10,12 \text { and } 14= \\
\text { NPK60 }=60 \mathrm{~kg} \mathrm{~N} \text { ha } \\
\text { PCMW20, PCMW40 } \\
100 \mathrm{~kg} \mathrm{~N} \mathrm{ha}^{-1} \text { of pure } \\
\mathrm{CMWFWSAU20,CI} \\
\text { and } 100 \mathrm{~kg} \mathrm{~N}^{-1} \text { of } \\
\text { SEM }=\text { Standard Err } \\
* * * \text { represent level o }\end{array}$ & $\begin{array}{l}\text { ree replicates } \\
\text { Veeks after planting } \\
\text { of NPK }(20: 10: 10) \text { fertilizer } \\
\text { PCMW60, PCMW80 and PCM } \\
\text { composted market waste. } \\
\text { WFWSAU40, CMWFWSAU60 } \\
\text { omposted market waste fortified } \\
\text { of Mean } \\
\text { significance at } p<0.001\end{array}$ & $\begin{array}{l}00=20,40 \\
\text { MWFWSA } \\
\text { th superph }\end{array}$ & $\begin{array}{l}0,80 \text { and } \\
80 \text { and } \mathrm{CMV} \\
\text { hate and ure }\end{array}$ & WSAU100 & $0,40,60,80$ & \\
\hline
\end{tabular}


Table 4: Effects of AM and fertilizers on the plant height $(\mathrm{cm})$.

\begin{tabular}{|c|c|c|c|c|c|c|}
\hline $\begin{array}{l}\text { Mycorrhiza } \\
\text { inoculation }\end{array}$ & $\begin{array}{l}\text { Fertilizers } \\
\text { application }\end{array}$ & 6 & 8 & 10 & 12 & 14 \\
\hline \multirow[t]{12}{*}{ With } & 0 & 42.20 & 84.40 & 86.10 & 105.10 & 133.10 \\
\hline & NPK & 101.67 & 149.17 & 165.17 & 185.17 & 222.17 \\
\hline & PCMW20 & 55.60 & 88.50 & 91.10 & 119.10 & 140.17 \\
\hline & PCMW40 & 83.37 & 110.17 & 128.10 & 150.10 & 180.17 \\
\hline & PCMW60 & 75.53 & 110.10 & 124.17 & 150.10 & 179.17 \\
\hline & PCMW80 & 72.67 & 100.17 & 117.03 & 125.10 & 155.10 \\
\hline & PCMW100 & 74.97 & 105.03 & 118.17 & 141.17 & 160.17 \\
\hline & CMWFWSAU20 & 61.30 & 94.20 & 99.10 & 120.10 & 146.10 \\
\hline & CMWFWSAU40 & 122.87 & 188.37 & 218.17 & 227.17 & 265.17 \\
\hline & CMWFWSAU60 & 111.17 & 151.07 & 177.17 & 195.17 & 244.17 \\
\hline & CMWFWSAU80 & 90.17 & 129.10 & 143.17 & 167.17 & 184.10 \\
\hline & CMWFWSAU100 & 94.40 & 133.37 & 150.17 & 170.17 & 201.17 \\
\hline \multirow[t]{12}{*}{ Without } & 0 & 20.20 & 40.30 & 48.10 & 56.10 & 95.10 \\
\hline & NPK & 94.67 & 140.17 & 151.17 & 178.17 & 204.03 \\
\hline & PCMW20 & 49.40 & 85.30 & 90.10 & 117.10 & 135.10 \\
\hline & PCMW40 & 63.60 & 98.30 & 108.10 & 123.10 & 147.10 \\
\hline & PCMW60 & 74.97 & 110.10 & 118.30 & 143.17 & 167.10 \\
\hline & PCMW80 & 70.67 & 99.30 & 112.17 & 125.10 & 153.17 \\
\hline & PCMW100 & 74.20 & 103.17 & 117.17 & 133.17 & 156.10 \\
\hline & CMWFWSAU20 & 60.60 & 90.10 & 95.50 & 119.17 & 145.10 \\
\hline & CMWFWSAU40 & 87.37 & 110.17 & 128.10 & 160.03 & 181.10 \\
\hline & CMWFWSAU60 & 99.37 & 142.17 & 155.17 & 180.17 & 220.17 \\
\hline & CMWFWSAU80 & 89.60 & 121.17 & 141.10 & 160.10 & 183.17 \\
\hline & CMWFWSAU100 & 90.67 & 131.17 & 149.17 & 170.17 & 187.17 \\
\hline \multicolumn{7}{|l|}{ SEM } \\
\hline \multicolumn{2}{|l|}{ Mycorrhiza (M) } & 0.4835 & 0.5730 & 0.5721 & 0.7617 & 0.7037 \\
\hline \multicolumn{2}{|l|}{ Fertilizers (F) } & 1.1844 & 1.4035 & 1.4013 & 1.8658 & 1.7237 \\
\hline \multicolumn{7}{|l|}{ Interaction } \\
\hline $\mathrm{M} \times \mathrm{F}$ & & 1.6751 & 1.9848 & 1.9817 & 2.6386 & 2.4377 \\
\hline \multicolumn{7}{|l|}{ ANOVA } \\
\hline M & & $* * *$ & $* * *$ & $* * *$ & $* * *$ & $* * *$ \\
\hline $\mathrm{F}$ & & $* * *$ & $* * *$ & $* * *$ & $* * *$ & $* * *$ \\
\hline \multicolumn{7}{|l|}{ Interaction } \\
\hline $\mathrm{M} \times \mathrm{F}$ & & $* * *$ & $* * *$ & $* * *$ & $* * *$ & $* * *$ \\
\hline $\begin{array}{l}\text { Values are means } \\
6,8,10,12 \text { and } 1 \\
\text { NPK60 }=60 \mathrm{~kg} \mathrm{I} \\
\text { PCMW20, PCM } \\
\text { of purely compos } \\
\text { CMWFWSAU20 } \\
\text { and } 100 \mathrm{~kg} \mathrm{~N} \text { ha }^{-1} \\
\text { SEM = Standard } \\
* * * \text { represent lev }\end{array}$ & $\begin{array}{l}\text { f three replicates } \\
=\text { Weeks after planting } \\
a^{-1} \text { of NPK }(20: 10: 10) \text { fer } \\
40, \text { PCMW60, PCMW80 } \\
\text { d market waste. } \\
\text { CMWFWSAU40, CMWF } \\
\text { f composted market waste } \\
\text { ror of Mean } \\
\text { of significance at } p<0.00\end{array}$ & $\begin{array}{l}\text { PCMW10 } \\
\text { AU60, CM } \\
\text { rtified with }\end{array}$ & $\begin{array}{l}0,40,60,8 \\
\text { WSAU } 80 \text { a } \\
\text { erphosphate }\end{array}$ & $\begin{array}{l}100 \mathrm{~kg} \mathrm{~N} \\
\text { MWFWS } \\
\text { urea }\end{array}$ & $00=20,4$ & \\
\hline
\end{tabular}


Table 5: Influence of AM and fertilizers on the colonization, bast and core yield.

\begin{tabular}{|c|c|c|c|c|}
\hline $\begin{array}{l}\text { Mycorrhiza } \\
\text { inoculation }\end{array}$ & Fertilizers application & Bast $\left(\right.$ g pot $\left.^{-1}\right)$ & Core $\left(\right.$ g pot $\left.^{-1}\right)$ & $\begin{array}{c}\text { Mycorrhizal } \\
\text { colonization }(\%)\end{array}$ \\
\hline \multirow[t]{12}{*}{ With } & 0 & 1.98 & 3.50 & 15.40 \\
\hline & NPK60 & 6.67 & 14.20 & 65.60 \\
\hline & PCMW20 & 2.27 & 5.24 & 17.30 \\
\hline & PCMW40 & 3.53 & 8.07 & 38.43 \\
\hline & PCMW60 & 3.36 & 7.85 & 29.50 \\
\hline & PCMW80 & 3.08 & 6.62 & 21.97 \\
\hline & PCMW100 & 3.36 & 7.30 & 25.37 \\
\hline & CMWFWSAU20 & 2.50 & 5.14 & 18.60 \\
\hline & CMWFWSAU40 & 8.75 & 17.09 & 73.10 \\
\hline & CMWFWSAU60 & 8.23 & 14.90 & 69.33 \\
\hline & CMWFWSAU80 & 4.26 & 9.57 & 52.63 \\
\hline & CMWFWSAU100 & 4.44 & 10.31 & 58.13 \\
\hline \multirow[t]{12}{*}{ Without } & 0 & 1.21 & 2.44 & 14.40 \\
\hline & NPK60 & 4.76 & 10.00 & 60.30 \\
\hline & PCMW20 & 2.13 & 4.15 & 16.47 \\
\hline & PCMW40 & 2.69 & 5.14 & 19.47 \\
\hline & PCMW60 & 3.24 & 7.00 & 27.23 \\
\hline & PCMW80 & 2.99 & 5.80 & 20.97 \\
\hline & PCMW100 & 3.17 & 6.15 & 22.67 \\
\hline & CMWFWSAU20 & 2.36 & 4.68 & 18.27 \\
\hline & CMWFWSAU40 & 3.74 & 8.51 & 44.43 \\
\hline & CMWFWSAU60 & 5.83 & 11.70 & 62.70 \\
\hline & CMWFWSAU80 & 3.94 & 8.68 & 49.93 \\
\hline & CMWFWSAU100 & 4.35 & 9.15 & 55.23 \\
\hline \multicolumn{5}{|l|}{ SEM } \\
\hline \multicolumn{2}{|l|}{$\begin{array}{l}\text { Mycorrniza } \\
\text { (M) }\end{array}$} & 0.0533 & 0.1281 & 0.5242 \\
\hline \multicolumn{2}{|l|}{ Fertilizers $(F)$} & 0.1307 & 0.3139 & 1.2841 \\
\hline \multicolumn{5}{|l|}{ Interaction } \\
\hline $\mathrm{M} \times \mathrm{F}$ & & 0.1848 & 0.4439 & 1.8160 \\
\hline \multicolumn{5}{|l|}{ ANOVA } \\
\hline M & & $* * *$ & $* * *$ & $* * *$ \\
\hline $\mathrm{F}$ & & $* * *$ & $* * *$ & $* * *$ \\
\hline \multicolumn{5}{|l|}{ Interaction } \\
\hline $\mathrm{M} \times \mathrm{F}$ & & $* * *$ & $* * *$ & $* * *$ \\
\hline \multicolumn{5}{|c|}{$\begin{array}{l}\text { Values are means of three replicates; } \\
\text { NPK60 = } 60 \mathrm{~kg} \mathrm{~N} \mathrm{ha}^{-1} \text { of NPK (20:10:10) fertilizer; } \\
\text { PCMW20, PCMW40, PCMW60, PCMW80 and PCMW100 }=20,40,60,80 \text { and } 100 \mathrm{~kg} \mathrm{~N} \mathrm{ha}^{-1} \\
\text { of purely composted market waste; } \\
\text { CMWFWSAU20, CMWFWSAU40, CMWFWSAU60, CMWFWSAU80 and CMWFWSAU100 = 20, 40, 60, } 80 \\
\text { and } 100 \mathrm{~kg} \mathrm{~N}^{-1} \text { of composted market waste fortified with superphosphate and urea; } \\
\text { SEM = Standard Error of Mean; } \\
* * * \text { represent level of significance at } \mathrm{p}<0.001 .\end{array}$} \\
\hline
\end{tabular}


Table 6: Residual effects of AM and fertilizers on the stem diameter (cm).

\begin{tabular}{|c|c|c|c|c|c|c|}
\hline $\begin{array}{l}\text { Mycorrhiza } \\
\text { inoculation }\end{array}$ & $\begin{array}{l}\text { Fertilizers } \\
\text { application }\end{array}$ & 6 & 8 & 10 & 12 & 14 \\
\hline \multirow[t]{12}{*}{ With } & 0 & 0.35 & 0.50 & 0.62 & 0.67 & 0.69 \\
\hline & NPK & 0.40 & 0.62 & 0.65 & 0.72 & 0.75 \\
\hline & PCMW20 & 0.44 & 0.65 & 0.70 & 0.78 & 0.81 \\
\hline & PCMW40 & 0.45 & 0.68 & 0.71 & 0.85 & 0.90 \\
\hline & PCMW60 & 0.50 & 0.73 & 0.77 & 0.88 & 0.91 \\
\hline & PCMW80 & 0.60 & 0.76 & 0.80 & 0.90 & 0.96 \\
\hline & PCMW100 & 0.62 & 0.90 & 1.00 & 1.02 & 1.08 \\
\hline & CMWFWSAU20 & 0.44 & 0.62 & 0.65 & 0.77 & 0.80 \\
\hline & CMWFWSAU40 & 0.45 & 0.66 & 0.71 & 0.82 & 0.87 \\
\hline & CMWFWSAU60 & 0.50 & 0.72 & 0.75 & 0.86 & 0.91 \\
\hline & CMWFWSAU80 & 0.60 & 0.75 & 0.80 & 0.90 & 0.96 \\
\hline & CMWFWSAU100 & 0.60 & 0.85 & 0.90 & 0.92 & 0.97 \\
\hline \multirow[t]{12}{*}{ Without } & 0 & 0.31 & 0.50 & 0.60 & 0.66 & 0.68 \\
\hline & NPK & 0.38 & 0.60 & 0.64 & 0.71 & 0.74 \\
\hline & PCMW20 & 0.44 & 0.63 & 0.70 & 0.78 & 0.80 \\
\hline & PCMW40 & 0.45 & 0.66 & 0.70 & 0.80 & 0.84 \\
\hline & PCMW60 & 0.46 & 0.70 & 0.75 & 0.86 & 0.91 \\
\hline & PCMW80 & 0.54 & 0.75 & 0.77 & 0.90 & 0.95 \\
\hline & PCMW100 & 0.60 & 0.79 & 0.80 & 0.92 & 0.96 \\
\hline & CMWFWSAU20 & 0.40 & 0.62 & 0.65 & 0.74 & 0.77 \\
\hline & CMWFWSAU40 & 0.45 & 0.65 & 0.70 & 0.79 & 0.83 \\
\hline & CMWFWSAU60 & 0.46 & 0.70 & 0.75 & 0.85 & 0.90 \\
\hline & CMWFWSAU80 & 0.52 & 0.74 & 0.77 & 0.90 & 0.93 \\
\hline & CMWFWSAU100 & 0.60 & 0.76 & 0.80 & 0.91 & 0.96 \\
\hline \multicolumn{7}{|l|}{ SEM } \\
\hline \multicolumn{2}{|l|}{ Mycorrhiza (M) } & 0.0019 & 0.0014 & 0.0025 & 0.0028 & 0.0044 \\
\hline \multicolumn{2}{|l|}{ Fertilizers (F) } & 0.0047 & 0.0033 & 0.0062 & 0.0069 & 0.0108 \\
\hline \multicolumn{7}{|l|}{ Interaction } \\
\hline$M \times F$ & & 0.0067 & 0.0047 & 0.0088 & 0.0097 & 0.0153 \\
\hline \multicolumn{7}{|l|}{ ANOVA } \\
\hline M & & $* * *$ & $* *$ & ns & ns & ns \\
\hline $\mathrm{F}$ & & $* * *$ & $* * *$ & $* * *$ & $* * *$ & $* * *$ \\
\hline \multicolumn{7}{|l|}{ Interaction } \\
\hline $\mathrm{M} \times \mathrm{F}$ & & $* * *$ & $* * *$ & $* * *$ & $* * *$ & $* * *$ \\
\hline $\begin{array}{l}\text { Values are means } \\
6,8,10,12 \text { and } 1 \\
\text { NPK60 } 60 \mathrm{~kg} \\
\text { PCMW20, PCMV } \\
\text { of purely compos } \\
\text { CMWFWSAU20 } \\
\text { and } 100 \mathrm{~kg} \mathrm{~N} \mathrm{ha}^{-1} \\
\text { SEM = Standard } \\
* * \text { and } * * * \text { repres } \\
\mathrm{ns}=\text { not significa }\end{array}$ & $\begin{array}{l}\text { hree replicates } \\
\text { Weeks after planting } \\
1 \text { of NPK (20:10:10) fertil } \\
\text { PCMW60, PCMW80 an } \\
\text { market waste. } \\
\text { AWFWSAU40, CMWFW } \\
\text { composted market waste } \mathrm{f} \\
\text { r of Mean } \\
\text { level of significance at } p\end{array}$ & $\begin{array}{l}\text { CMW100 } \\
\text { U60, CMY } \\
\text { fied with s } \\
01 \text { and } 0.0\end{array}$ & $\begin{array}{l}40,60,80 \\
\text { SAU80 and } \\
\text { hosphate a } \\
\text { pectively }\end{array}$ & $\begin{array}{l}100 \mathrm{~kg} \mathrm{~N} \mathrm{~h} \\
\text { IWFWSAI } \\
\text { Irea }\end{array}$ & $=20,40$ & \\
\hline
\end{tabular}


Table 7: Residual effects of AM and fertilizers on the plant height (cm).

\begin{tabular}{|c|c|c|c|c|c|c|}
\hline $\begin{array}{l}\text { Mycorrhiza } \\
\text { inoculation }\end{array}$ & $\begin{array}{l}\text { Fertilizers } \\
\text { application }\end{array}$ & 6 & 8 & 10 & 12 & 14 \\
\hline \multirow[t]{12}{*}{ With } & 0 & 37.17 & 56.10 & 65.10 & 70.10 & 89.00 \\
\hline & NPK & 47.17 & 78.17 & 92.10 & 98.10 & 108.37 \\
\hline & PCMW20 & 54.10 & 95.10 & 111.10 & 117.10 & 126.70 \\
\hline & PCMW40 & 58.10 & 102.17 & 117.17 & 130.10 & 142.43 \\
\hline & PCMW60 & 62.10 & 111.10 & 126.10 & 142.17 & 158.37 \\
\hline & PCMW80 & 68.10 & 114.17 & 137.17 & 166.17 & 177.43 \\
\hline & PCMW100 & 80.10 & 137.10 & 160.17 & 193.17 & 209.43 \\
\hline & CMWFWSAU20 & 50.10 & 88.10 & 98.17 & 107.17 & 113.43 \\
\hline & CMWFWSAU40 & 57.17 & 100.17 & 117.10 & 130.10 & 141.37 \\
\hline & CMWFWSAU60 & 60.10 & 108.10 & 120.17 & 142.10 & 151.77 \\
\hline & CMWFWSAU80 & 63.17 & 113.50 & 136.17 & 162.17 & 176.10 \\
\hline & CMWFWSAU100 & 77.10 & 130.17 & 153.17 & 180.17 & 196.43 \\
\hline \multirow[t]{12}{*}{ Without } & 0 & 30.17 & 54.10 & 60.10 & 65.10 & 79.00 \\
\hline & NPK & 45.10 & 75.17 & 82.10 & 95.10 & 104.70 \\
\hline & PCMW20 & 52.17 & 90.10 & 100.10 & 112.10 & 123.37 \\
\hline & PCMW40 & 54.17 & 100.17 & 115.17 & 120.17 & 130.37 \\
\hline & PCMW60 & 60.10 & 103.10 & 120.10 & 134.10 & 148.03 \\
\hline & PCMW80 & 63.10 & 113.10 & 130.17 & 158.17 & 168.43 \\
\hline & PCMW100 & 74.17 & 122.17 & 138.10 & 178.17 & 192.10 \\
\hline & CMWFWSAU20 & 48.10 & 80.10 & 95.17 & 102.17 & 111.00 \\
\hline & CMWFWSAU40 & 54.17 & 97.10 & 113.10 & 120.10 & 129.77 \\
\hline & CMWFWSAU60 & 58.17 & 102.17 & 117.17 & 132.17 & 144.03 \\
\hline & CMWFWSAU80 & 62.17 & 112.17 & 130.10 & 148.17 & 159.43 \\
\hline & CMWFWSAU100 & 73.17 & 120.17 & 137.17 & 172.10 & 188.37 \\
\hline \multicolumn{7}{|l|}{ SEM } \\
\hline \multicolumn{7}{|l|}{ Mycorrhiza } \\
\hline (M) & & 0.8222 & 0.6876 & 0.8413 & 0.7538 & 1.1729 \\
\hline Fertilizers $(\mathrm{F})$ & & 2.0139 & 1.6843 & 2.0607 & 1.8464 & 2.8731 \\
\hline \multicolumn{7}{|l|}{ Interaction } \\
\hline$M \times F$ & & 2.8481 & 2.3820 & 2.9143 & 2.6113 & 4.0631 \\
\hline \multicolumn{7}{|l|}{ ANOVA } \\
\hline M & & $* * *$ & $* * *$ & $* * *$ & $* * *$ & $* * *$ \\
\hline $\mathrm{F}$ & & $* * *$ & $* * *$ & $* * *$ & $* * *$ & $* * *$ \\
\hline \multicolumn{7}{|l|}{ Interaction } \\
\hline$M \times F$ & & $*$ & $* * *$ & $* * *$ & $* *$ & ns \\
\hline $\begin{array}{l}\text { Values are me } \\
6,8,10,12 \text { an } \\
\text { NPK60 }=60 \mathrm{k} \\
\text { PCMW20, PC } \\
\text { composted ma } \\
\text { CMWFWSAl } \\
\text { and } 100 \mathrm{~kg} \mathrm{~N} \\
\text { SEM }=\text { Stand } \\
*, * * \text { and } * * * \\
\text { ns }=\text { not signif }\end{array}$ & $\begin{array}{l}\text { S of three replicates } \\
4=\text { Weeks after planting } \\
\mathrm{N} \mathrm{ha}^{-1} \text { of NPK }(20: 10: 10) \\
\text { IW40, PCMW60, PCMV } \\
\text { et waste. } \\
\text {, CMWFWSAU40, CMV } \\
1 \text { of composted market wa } \\
\text { Error of Mean } \\
\text { oresent level of significan }\end{array}$ & $\begin{array}{l}\text { rtilizer } \\
30 \text { and PC } \\
\text { FWSAU60 } \\
\text { te fortified } \\
\text { at } \mathrm{p}<0.0\end{array}$ & $\begin{array}{l}\text { W100 }=2 \\
\text { CMWFWS } \\
\text { ith superph } \\
0.01 \text { and } 0 .\end{array}$ & $\begin{array}{l}40,60,80 \\
\text { J80 and CM } \\
\text { phate and ur } \\
1 \text { respectivel }\end{array}$ & FWSAU100 & $\begin{array}{l}a^{-1} \text { of purely } \\
20,40,60,80\end{array}$ \\
\hline
\end{tabular}


Table 8: Residual effect of AM and fertilizers on the colonization, bast and core yield

\begin{tabular}{|c|c|c|c|c|}
\hline $\begin{array}{l}\text { Mycorrhiza } \\
\text { inoculation }\end{array}$ & Fertilizers application & Bast $\left(\right.$ g pot $\left.^{-1}\right)$ & Core $\left(\right.$ g pot $\left.^{-1}\right)$ & $\begin{array}{c}\text { Mycorrhizal } \\
\text { colonization }(\%)\end{array}$ \\
\hline \multirow[t]{12}{*}{ With } & 0 & 1.46 & 3.31 & 12.33 \\
\hline & NPK60 & 1.84 & 3.95 & 13.50 \\
\hline & PCMW20 & 2.14 & 4.33 & 16.10 \\
\hline & PCMW40 & 2.38 & 5.19 & 20.53 \\
\hline & PCMW60 & 2.67 & 6.27 & 30.27 \\
\hline & PCMW80 & 3.32 & 6.69 & 42.53 \\
\hline & PCMW100 & 5.27 & 10.55 & 56.10 \\
\hline & CMWFWSAU20 & 2.05 & 4.07 & 14.47 \\
\hline & CMWFWSAU40 & 2.33 & 4.77 & 18.70 \\
\hline & CMWFWSAU60 & 2.56 & 5.80 & 27.17 \\
\hline & CMWFWSAU80 & 2.97 & 6.61 & 39.10 \\
\hline & CMWFWSAU100 & 4.68 & 8.19 & 51.57 \\
\hline \multirow[t]{12}{*}{ Without } & 0 & 1.32 & 2.71 & 11.50 \\
\hline & NPK60 & 1.71 & 3.79 & 12.97 \\
\hline & PCMW20 & 1.96 & 3.95 & 15.30 \\
\hline & PCMW40 & 2.14 & 4.70 & 17.63 \\
\hline & PCMW60 & 2.40 & 5.60 & 23.90 \\
\hline & PCMW80 & 2.75 & 6.52 & 34.27 \\
\hline & PCMW100 & 4.26 & 7.00 & 47.97 \\
\hline & CMWFWSAU20 & 1.85 & 4.02 & 13.53 \\
\hline & CMWFWSAU40 & 2.10 & 4.49 & 17.10 \\
\hline & CMWFWSAU60 & 2.33 & 5.42 & 22.47 \\
\hline & CMWFWSAU80 & 2.67 & 6.44 & 33.27 \\
\hline & OM100 & 3.77 & 6.76 & 45.10 \\
\hline \multicolumn{5}{|l|}{ SEM } \\
\hline Mycorrhiza (M) & & 0.0460 & 0.0652 & 0.3965 \\
\hline Fertilizers $(\mathrm{F})$ & & 0.1127 & 0.1597 & 0.9713 \\
\hline \multicolumn{5}{|l|}{ Interaction } \\
\hline $\mathrm{M} \times \mathrm{F}$ & & 0.1593 & 0.2259 & 1.3736 \\
\hline \multicolumn{5}{|l|}{ ANOVA } \\
\hline M & & $* * *$ & $* * *$ & $* * *$ \\
\hline $\mathrm{F}$ & & $* * *$ & $* * *$ & $* * *$ \\
\hline \multicolumn{5}{|l|}{ Interaction } \\
\hline $\mathrm{M} \times \mathrm{F}$ & & ns & $* * *$ & $*$ \\
\hline $\begin{array}{l}\text { Values are means } \\
\text { NPK60 }=60 \mathrm{~kg} \mathrm{~N} \\
\text { PCMW20, PCMW } \\
\text { of purely compost } \\
\text { CMWFWSAU20, } \\
\text { and } 100 \mathrm{~kg} \mathrm{~N} \mathrm{ha}^{-1} \\
\text { SEM }=\text { Standard I } \\
* \text { and } * * * \text { represen } \\
\mathrm{ns}=\text { not significan }\end{array}$ & $\begin{array}{l}\text { three replicates } \\
-1 \text { of NPK (20:10:10) fertilizer } \\
\text {, PCMW60, PCMW } 80 \text { and PCI } \\
\text { market waste. } \\
\text { MWFWSAU40, CMWFWSAU } \\
\text { composted market waste fortifi } \\
\text { or of Mean } \\
\text { evel of significance at } \mathrm{p}<0.05\end{array}$ & $\begin{array}{l}\text { W100 }=20,40,60, \\
\text { CMWFWSAU80 } \\
\text { with superphospha } \\
\text { d } 0.001 \text { respectively }\end{array}$ & $\begin{array}{l}0 \text { and } 100 \mathrm{~kg} \mathrm{~N} \mathrm{ha}^{-1} \\
\text { and urea }\end{array}$ & $=20,40,60,80$ \\
\hline
\end{tabular}




\section{DISCUSSION}

The soil used for this study was low in nutrients compared to the soil fertility rating classes in Nigerian (FPDD, 1990). Low fertility status of the soil might be due to the continuous cultivation of the land with continuous application of inorganic fertilizer in the past. The infective propagule density was low, less than 140 infective propagules per $100 \mathrm{~g}$ soil (Sieverding, 1991).

Tiannug 1 showed a higher degree of responsiveness to mycorrhizal inoculation. Variation in response to mycorrhizal inoculation has been obtained in different species and genotypes of other crops (Dare et al., 2008). However, inoculation increased the growth, AM colonization, bast and core yield of Tiannug 1 compared to the non-inoculation counterpart. These better growth exhibited by the inoculated might be due to a better uptake of nutrients, which in turn can be directly attributed to AM inoculation. This might also be due to the effectiveness of Glomus mosseae inoculated to absorb plant nutrient from soil solution for the growth and yield of the plant. Increase in the levels of the compost applied with mycorrhizal inoculation significantly $(\mathrm{p}$ $<0.001$ ) increased mycorrhizal colonization, bast and core yield from 20 to $40 \mathrm{~kg} \mathrm{~N}^{-1}$ of both PCMW and CMWFWSAU. High amount of soil available $\mathrm{P}$ and total $\mathrm{N}$ may lower AM colonization (Treseder and Allen, 2002; Johnson et al., 2003).

On the residual effect of AM inoculation and compost application, percentage AM colonization, growth and yield parameters increased from $20 \mathrm{~kg} \mathrm{~N}^{-1}{ }^{-1}$ to 100 $\mathrm{kg} \mathrm{N} \mathrm{ha}{ }^{-1}$ of both PCMW and CMWFWSAU. PCMW had better yields than CMWFWSAU. This might be due to the gradual release of nutrients by the PCMW which make it to have better residual effect among the compost used.

\section{Conclusion}

Tiannug 1 was colonized by arbuscular mycorrhiza. Organic base fertilizer had higher percentage mycorrhizal colonization than the recommended rate of NPK (20: 10: 10) fertilizer. Percentage root colonization decreased as the nitrogen level increased. The higher the percentage root colonization, the higher were the growth (stem girth and plant height) and yield (bast and core) parameters. Inoculated Tiannug 1 at $40 \mathrm{~kg} \mathrm{~N} \mathrm{ha}^{-1}$ of CMWFWSAU had significantly higher mycorrhizal colonization, bast and core yield. On the residual effects of fertilizers application and mycorrhizal inoculation, inoculated Tiannug 1 of PCMW had significantly higher mycorrhizal colonization, bast and core yield. For sustainable kenaf production, $40 \mathrm{~kg} \mathrm{~N} \mathrm{ha}^{-1}$ of organic fertilizer along with arbuscular mycorrhiza is recommended. However, there is need to manage indigenous mycorrhiza and organic matter content of the soil to reduce input of chemical fertilizer through the application of organic material.

\section{REFERENCES}

Atayese MO, Awotoye OO, Osonubi O, Mulongoy K. 1993. Comparisons of the influence of vesicular - arbuscular mycorrhizal on the productivity of hedgerow woody legumes and cassava at the top and the base of a hillslope in alley cropping systems. Biology and Fertility of Soils, 16: 198 - 204.

Bada BS, Kalejaiye ST. 2010. Response of kenaf (Hibiscus cannabinus L.) grown in different soil textures and lead concentrations. Research Journal of Agriculture and Biological Sciences, 6(5): 659 - 664.

Bada BS, Olatunde AK, Taiwo DA. 2012. Heavy metal content of soil and jute mallow collected from organic and 
inorganic farms. Journal of Agriculture, Forestry and the Social Sciences, 10(2): $211-217$.

Bada BS, Raji KA. 2010. Phytoremediation potential of kenaf (Hibiscus cannabinus L.) grown in different soil textures and cadmium concentrations. African Journal of Environmental Science and Technology, 4(5): $160-168$.

Bada BS, Umunnakwe CF. 2011. Phytoremediation potential of kenaf (Hibiscus cannabinus linn.) under different soil $\mathrm{pH}$ and copper concentrations. Journal of Science Research, 10(2): 160 - 166.

Bert N. 2002. Kenaf fibres. Presentation of the $5^{\text {th }}$ Annual Conference of the American Kenaf Society, Memphis TN Nov $7-9$, pp. $15-22$.

Carling DE, Riechle WG, Brown MF, Johnson DR. 1978. Effect of a vesiculararbuscular mycorrhiza fungus on nitrogen reductase and nitrogenase activities in nodulating and non-nodulating soybeans. Phytopathology, 68: 1590 - 1596.

Cheng Z. 2001. Kenaf research products and applications in Japan. Plant Fibres and Products, 23(3): 16 - 24.

Chopra SL, Kanwar JS. 1999. Analytical Agricultural Chemistry. Kalyani publishers: New Delhi; 152 - 195.

Clark RB, Zeto SK. 2000. Mineral acquisition by arbuscular mycorrhizal plants. Journal of Plant Nutrition, 23(7): 867 - 902.

Dare MO, Abaidoo RC, Fagbola O, Asiedu R. 2008. Genetic variation and genotype environment interaction in yams (Dioscorea Spp.) for root colonization by arbuscular mycorrhiza. Journal of Food, Agriculture and Environment, 6(2): 227 233.

FPDD (Fertilizer Procurement and Distribution Division). 1990. Literature review on soil fertility investigations in Nigeria. Food Chemistry, 78: 63 - 68.

Giovanneti M, Mosse B. 1980. An evaluation of techniques to measures vesicular arbuscular infections in roots. New Phytologist, 184: 489 - 500.

Hodge A. 2003. Plant nitrogen capture from organic matter as affected by spatial dispersion, interspecific competition and mycorrhizal colonization. New Phytologist, 157(2): 303-314.

IART (Institute of Agricultural Research and Training). 2010. Guide on kenaf production and processing. IART, farmers' guide series 1 no. 1, 1 - 7 .

Ibiremo OS, Fagbola O. 2008. Effect of phosphate fertilizers and arbuscular mycorrhizal fungi inoculation on the growth of cashew seedlings in two soils in Nigeria. Nigerian Journal of Soil Science, 18: 138 - 146.

Johnson NC, Rowland DL, Corkidiki L, Egerton-Warburton LM, Allen EB. 2003. Nitrogen enrichment alters mycorrhizal allocation at five mesic to semiarid grasslands. Ecology, 84: 1895 - 1908.

Liu AM. 2003. Making pulp and paper from kenaf. Available in http:www. chinaconsultinginc.com/paperpulp.htm [03 July 2008].

Omueti JAI, Sridhar MKC, Adeoye GO, Bamiro O, Fadere DA. 2000. Organic fertilizer use in Nigeria: Our experience. In Agronomy in Nigeria, Akoroda MO (ed). University of Ibadan, Ibadan, Nigeria; 208 - 215.

Porter WM. 1979. The most probable numbers method for enumerating infectiveness of VAM fungi in soil. Australian Journal of Soil Research, 17: $515-519$.

Sameshima K. 2000. Improvement of kenaf core oil absorption property by heat 
treatment at $200-500{ }^{\circ} \mathrm{C}$. Proceedings of the $3^{\text {rd }}$ annual American Kenaf Society Conference, Corpus Christi, TX, Feb, p. $64-72$.

Sieverding E. 1991. Vesicular-arbuscular mycorrhiza managmenet in tropical agrosystems. Deutsche GesellsChaft fur Technische Zusammenarbeit (GTZ) $\mathrm{GmbH}$, Federal Republic of Germany, p. 295.
Treseder KK, Allen MF. 2002. Direct nitrogen and phosphorus limitation of arbuscular mycorrhizal fungi: a model and field test. New Phytologist, 155: 507 - 515.

Wilson FD. 2003. Kenaf history and botany. Economic Botany, 18: 80 - 91.

Zhang T. 2003. Improvement of kenaf yarn for apparel application. Master's thesis. Louisiana State Univ. US, 87p. 